\title{
NMR spectroscopy and perfusion of mammalian cells using surface microprobes
}

\author{
Klaus Ehrmann, ${ }^{a}$ Kristopher Pataky, ${ }^{a}$ Matthieu Stettler, ${ }^{b}$ Florian Maria Wurm, ${ }^{b}$ Jürgen Brugger, ${ }^{a}$ \\ Pierre-André Besse ${ }^{* a}$ and Radivoje Popovic ${ }^{a}$
}

Received 12th September 2006, Accepted 5th December 2006

First published as an Advance Article on the web 18th January 2007

DOI: $10.1039 / \mathrm{b} 613240 \mathrm{e}$

NMR spectra of mammalian cells are taken using surface microprobes that are based on microfabricated planar coils. The surface microprobe resembles a miniaturized Petri dish commonly used in biological research. The diameter of the planar coils is $1 \mathrm{~mm}$. Chinese Hamster Ovaries are immobilized in a uniform layer on the microprobe surface or patterned by an ink-jet printer in the centre of the microcoil, where the rf-field of the planar microcoil is most uniform. The acquired NMR spectra show the prevalent metabolites found in mammalian cells. The volumes of the detected samples range from $25 \mathrm{~nL}$ to $1 \mathrm{~nL}$ (or 50000 to 1800 cells). With an extended set-up that provides fluid inlets and outlets to the microprobe, the cells can be perfused within the NMR-magnet while constantly taking NMR spectra. Perfusion of the cells opens the way to increased cell viability for long acquisitions or to analysis of the cells' response to environmental change.

\section{Introduction}

Nuclear magnetic resonance is a very information-rich and non-destructive analysis technique and is therefore well-suited to the analysis of living samples. Downscaling of the NMRprobes allows a significant increase in the sensitivity when having mass-limited samples. ${ }^{1}$

Most microfabricated NMR-probes are based on planar coils combined with microfluidic channels to confine the sample. $^{2,3}$ In a previous contribution, we characterized the performance of our surface microprobe and demonstrated a dramatic improvement of the spin excitation uniformity by patterning artificial vesicles in the centre of the coil, where the rf-field is uniform. ${ }^{4}$ We have demonstrated the feasibility of taking spectra of cells confined in a micro-channel with microfabricated Helmholtz coils as well. ${ }^{5}$ However, with such a 3D confined structure, cell perfusion was not feasible due to the excessive pressure required to exchange the liquids.

In this contribution we use a surface microprobe, offering an open environment to the mammalian cells, for the NMR spectroscopy and the perfusion. In a first experiment spectra of cells are taken that are uniformly distributed on the microprobe surface. In a second experiment an inkjet printer is used to pattern the cells in the centre of the micro-coil. During long acquisitions, perfusion of the cells with fresh medium is necessary to maintain stable environmental conditions and cell viability. In a third experiment the perfusion of mammalian cells and simultaneous NMR acquisition are demonstrated.

\footnotetext{
${ }^{a}$ Microsystems Laboratory, EPFL-Ecole Polytechnique Fédérale de Lausanne, CH-1015 Lausanne, Switzerland. E-mail: pierreandre.besse@epfl.ch; Fax:+41216936670; Tel: +41216938047

${ }^{b}$ Laboratory of Cellular Biotechnology, EPFL-Ecole Polytechnique

Fédérale de Lausanne, CH-1015 Lausanne, Switzerland
}

\section{Experimental}

\section{Spectroscopy set-up}

The planar surface micro-coils are batch fabricated on 4-in glass wafers in five photolithographic steps with two copper levels, an SU-8 isolation layer in between, and an SU-8 encapsulation layer. The process is described in detail by Ehrmann et al. ${ }^{4}$ In order to resemble a Petri dish or a well plate, a PMMA cylinder, which serves as sample container, is glued on top of the SU-8 surface of the microcoil. The microcoil chip is bonded to a PCB, where the resonant circuit is implemented such that the microprobe can be connected directly to the preamplifier of a conventional NMR spectrometer.

The sample perfusion has to be done within the NMRmagnet during acquisition. Fig. 1 shows the extended surface microprobe for sample perfusion. A channel milled in a PMMA plate passes on top of the sample container. The channel is connected to tubes by microfluidic fittings (Upchurch Scientific). The tube at the inlet is connected to a pump (L-6200A, Hitachi). The tube at the outlet leads to a waste receptacle.

\section{Cell preparation}

CHO DG44 cells, which are adapted to growing in a serumfree suspension, are cultured in ProCHO5 CDM medium (Bio Whittaker Inc.). For taking ${ }^{1} \mathrm{H}$ spectra of $\mathrm{CHO}$ cells, the cell medium is exchanged with deuterated PBS (phosphor buffered saline). The cell medium exchange is done by several cycles of centrifuging the cells in their solution, removing the supernatant and re-suspending the cells in deuterated PBS. 
(a)

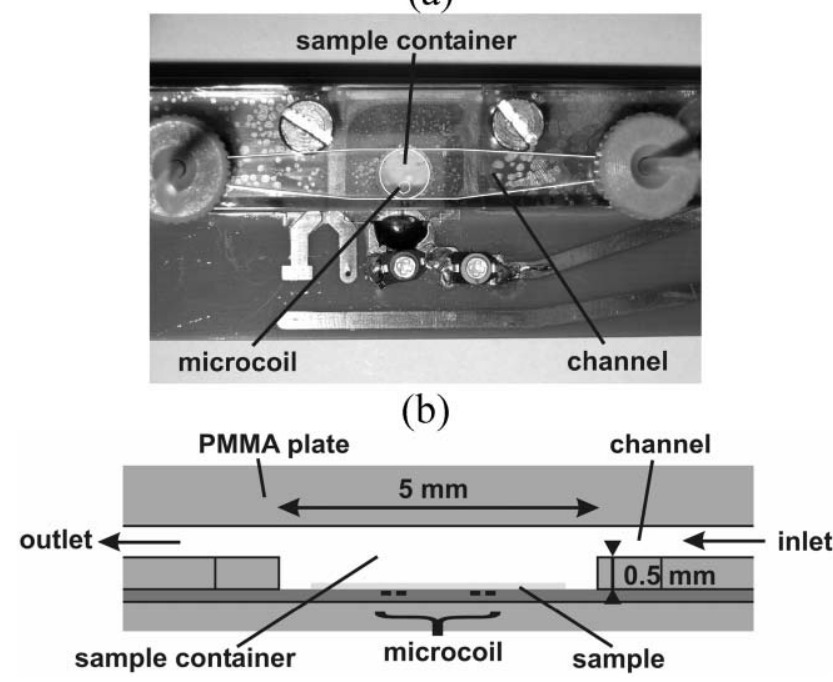

Fig. 1 Picture (a) and drawing (b) of extended surface microprobe for sample perfusion within the NMR-magnet during analysis.

\section{Results and discussion}

\section{Spectroscopy of mammalian cells}

The CHO DG44 cells are loaded onto the surface microprobe by dispensing them in their medium into the sample container with a pipet.

Keeping the microprobe horizontal, the cells concentrate on the surface by sedimentation. The final cell density on the surface can be controlled by the cell density of the medium. In the experiment described a cell density of 50 million cells $\mathrm{mL}^{-1}$ is chosen, which gives a double layer of mammalian cells on the probe surface. After sedimentation the cells immobilize on the SU-8 surface. After dispensing the cells, the sample container is directly closed with an adhesive film (Falcon, BD). Placing the probe into the NMR magnet for analysis turns the surface vertical. In spite of this, the cells stay attached to the surface for weeks.

Fig. 2 shows the ${ }^{1} \mathrm{H}$ spectrum acquired after 4096 scans. The total acquisition time is $4 \mathrm{~h}$. Water suppression by presaturation is applied to reduce the peak of the water residues in deuterated PBS. The peaks of the spectrum are assigned

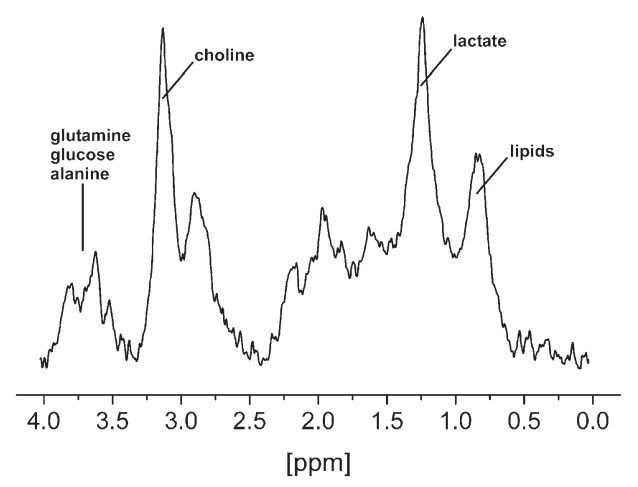

Fig. 2 Spectrum of $\mathrm{CHO}$ cells that are uniformly immobilized on the microprobe surface. according to published cell spectra and chemical shifts of metabolites. ${ }^{6-8}$ The number of detected cells is about 50000 and the detected cell volume is about $25 \mathrm{~nL}$. Owing to the lack of an optical access in the NMR set-up, the viability of the cells during the acquisition cannot be monitored.

In a previous contribution, lipid vesicles were patterned in the coil centre by aligned microcontact printing to improve the spin excitation uniformity. ${ }^{4}$ Microcontact printing can also be used when patterning living cells. Then, however, an extracellular matrix would be printed onto a surface. ${ }^{9}$ Here, inkjet printing is used to pattern the cells in the regions of uniform $\mathrm{B}_{1}$-field on the microprobe surface. Inkjet printing has the advantage of being very rapid and inexpensive, especially when commercial inkjet printers can be used. ${ }^{10}$

A piezo-electrically driven inkjet printer with a nozzle that has a $50 \mu \mathrm{m}$ aperture (MD-K-140, Microdrop Technologies) is used for patterning cells on the microprobe surface. The microprobe is positioned on automated stages that allow lateral movement for the alignment of the printer with the microcoil. The alignment is carried out optically with a CCD camera (Toshiba-Teli Co.).

$\mathrm{CHO}$ cells at a density of 100 million cells $\mathrm{mL}^{-1}$ are used. 300 droplets, equivalent to $18 \mathrm{~nL}$ of suspension or 1800 cells are printed onto the centre of the microcoil. After the printing, the sample container is filled with deuterated PBS and closed with an adhesive tape. Fig. 3 (a) shows $\mathrm{CHO}$ cells that were patterned and then analyzed by NMR spectroscopy. Because of the dispersion at the nozzle and container filling some cells are driven out of the microcoil centre.

A ${ }^{1} \mathrm{H}$ spectrum is acquired after 4096 scans (Fig. 3(b)) using the same acquisition parameters as in the non-patterned

(a)

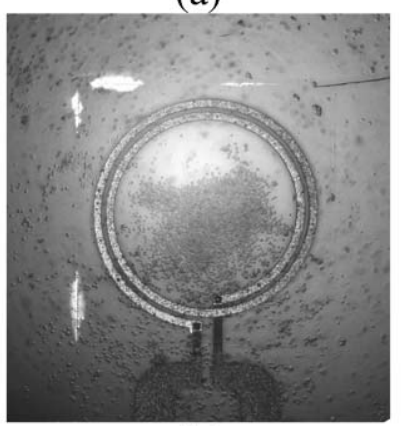

(b)

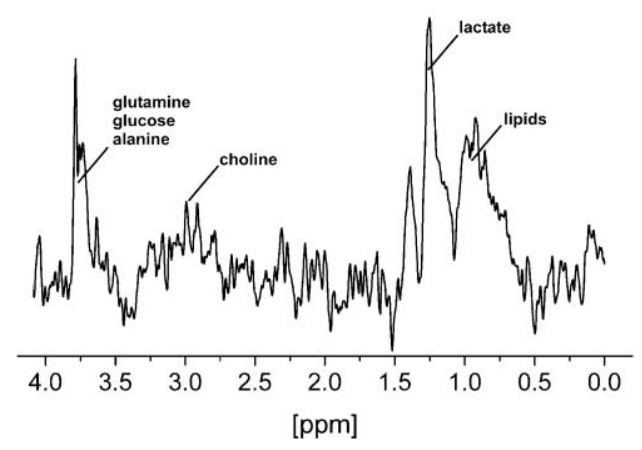

Fig. 3 Picture of $\mathrm{CHO}$ cells that are printed in the centre of the microprobe surface (a) and the spectrum taken of these cells (b). 


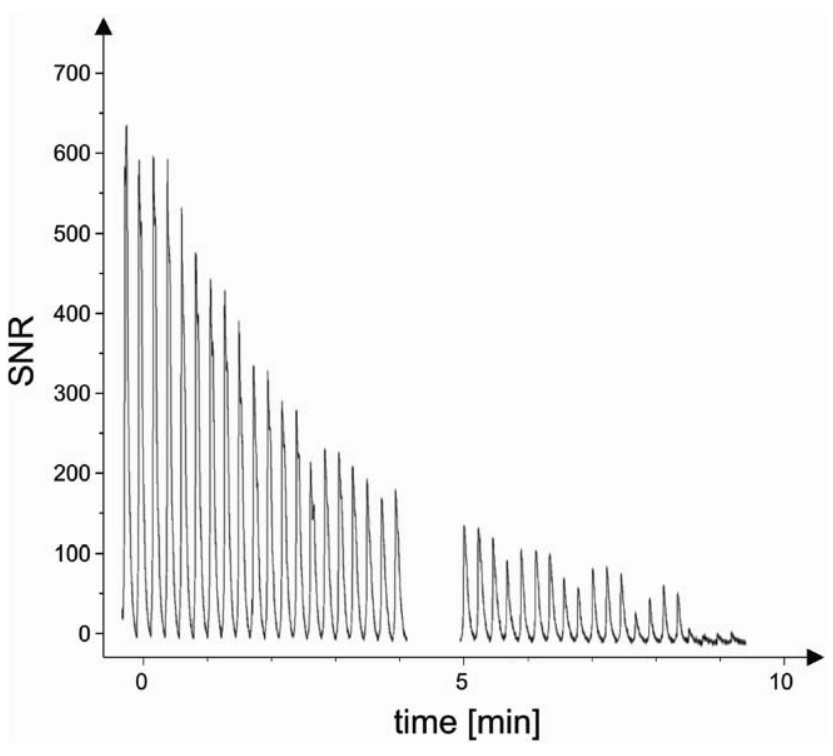

Fig. 4 NMR-spectra of decreasing DMSO peak during perfusion.

experiment. The SNR, however, is much lower, which can be explained by the smaller detected cell volume of only $1 \mathrm{~nL}$ compared with $25 \mathrm{~nL}$ in the former case. The peak of the glutamine, glucose and alanine molecules is comparatively high; the choline peak has almost disappeared. Since no investigations on the biological activities of the cell metabolites have been conducted, the cause of the altered signals cannot be explained. Furthermore, cell viability is not guaranteed after $4 \mathrm{~h}$ of acquisition. For such long acquisitions, sample perfusion would be necessary to control the environmental conditions and assure viability of the cells.

\section{Perfusion of mammalian cells}

The feasibility of sample perfusion is demonstrated by exchanging an initial cell medium that contains $10 \%$ of DMSO (dimethyl sulfoxide) for a plain cell medium while having cells immobilized on the surface of the microprobe. The disappearance of DMSO is monitored by continually acquiring NMR spectra during the perfusion. The ${ }^{1} \mathrm{H}$ spectrum of DMSO has one distinct peak far away from the water peak, which allows measuring even very small quantities of DMSO.

CHO DG44 cells in their grow-medium are used for the experiment. The cell density is increased to 20 million cells $\mathrm{mL}^{-1}$ by centrifugation. The $10 \% \mathrm{DMSO}$ is added afterwards.

The cells in their medium are injected into the tubes and pumped into the microprobe. The microprobe is placed in a horizontal position such that the cells deposit onto the coil surface. After $1 \mathrm{~h}$, the cells are immobilized on the surface and the probe is inserted into the NMR-magnet. Plain medium is introduced into the inlet tube and pumped into the microprobe at a pumping rate of $0.1 \mathrm{~mL} \mathrm{~min}^{-1}$. At the same time, an NMR acquisition cycle is started. Fig. 4 shows the SNR of the DMSO peak dependent on the time of the acquisition. The plain medium arrives in the sample container at time 0. From then on the DMSO signal is decreasing to the same extent as the DMSO is purged out of the microprobe.

Observing the cells before and after the perfusion with a microscope shows that the perfusion flow does not significantly influence the cell positions. Only very few cells are washed away.

\section{Conclusions}

NMR surface-microprobes that imitate Petri-dishes have been developed for the analysis of cells. NMR-spectra of CHO cells that are uniformly immobilized on the surface-microprobe or printed in the centre of the microprobe using an inkjet printer have been successfully taken. The spectra show the peaks of choline, lactate and lipids. These results demonstrate the feasibility of carrying out NMR spectroscopy of cells with microfabricated NMR-probes.

An extended set-up for perfusion of the cells within the NMR-magnet is presented. The liquid exchange is monitored in real time by NMR spectroscopy and does not affect the cells, which are immobilized on the microprobe surface. The possibility of perfusing the sample in the NMR-magnet opens the way to using the microprobe for long acquisitions of living cells under stable environmental conditions and increased viability.

\section{References}

1 A. G. Webb, Prog. Nucl. Magn. Reson. Spectrosc., 1997, 31, 1 .

2 C. Massin, F. Vincent, A. Homsy, K. Ehrmann, G. Boero, P. A. Besse, A. Daridon, E. Verpoorte, N. F. De Rooij and R. S. Popovic, J. Magn. Reson., 2003, 164, 242.

3 J. D. Trumbull, I. K. Glasgow, D. J. Beebe and R. L. Magin, IEEE Trans. Biomed. Eng., 2000, 47, 3.

4 K. Ehrmann, M. Gersbach, P. Pascoal, F. Vincent, C. Massin, D. Stamou, P. A. Besse, H. Vogel and R. S. Popovic, J. Magn. Reson., 2006, 178, 96.

5 K. Ehrmann, N. Saillen, M. Stettler, F. Vincent, M. Jordan, F. M. Wurm, P.-A. Besse and R. S. Popovic, Lab Chip, 2006, DOI: $10.1039 / \mathrm{b} 614044 \mathrm{k}$

6 P. F. Agris and I. D. Campbell, Science, 1982, 216, 1325.

7 V. Govindaraju, K. Young and A. A. Maudsley, NMR Biomed., 2000, 13, 129.

8 R. S. Kane, S. Takayama, E. Ostuni, D. E. Ingber and G. M. Whitesides, Biomaterials, 1999, 20, 2363.

9 E. Ostuni, R. Kane, C. S. Chen, D. E. Ingber and G. M. Whitesides, Langmuir, 2000, 16, 7811.

10 E. A. Roth, T. Xu, M. Das, C. Gregory, J. J. Hickman and T. Boland, Biomaterials, 2004, 25, 3707. 